\title{
Observation of an electrically tunable band gap in trilayer graphene
}

\author{
Chun Hung Lui ${ }^{1}$, Zhiqiang Li ${ }^{1}$, Kin Fai Mak ${ }^{1}$, Emmanuele Cappelluti ${ }^{2,3}$ and Tony F. Heinz ${ }^{1 \star}$
}

\begin{abstract}
A striking feature of bilayer graphene is the induction of a significant band gap in the electronic states by the application of a perpendicular electric field ${ }^{1-7}$. Thicker graphene layers are also highly attractive materials. The ability to produce a band gap in these systems is of great fundamental and practical interest. Both experimental ${ }^{8}$ and theoretical ${ }^{9-16}$ investigations of graphene trilayers with the typical ABA layer stacking have, however, revealed the lack of any appreciable induced gap. Here we contrast this behaviour with that exhibited by graphene trilayers with $A B C$ crystallographic stacking. The symmetry of this structure is similar to that of AB-stacked graphene bilayers and, as shown by infrared conductivity measurements, permits a large band gap to be formed by an applied electric field. Our results demonstrate the critical and hitherto neglected role of the crystallographic stacking sequence on the induction of a band gap in few-layer graphene.
\end{abstract}

Producing a controlled and tunable band gap in graphene is a topic of central importance ${ }^{1-7,17,18}$. In addition to the intrinsic interest of altering the electronic properties of materials, the availability of an adjustable band gap opens up the possibility of a much wider range of applications for graphene in electronics and photonics. Both single- and few-layer graphene in their unperturbed state lack a band gap ${ }^{19,20}$. However, few-layer graphene materials under the application of a symmetry-lowering perpendicular electric field may exhibit an induced gap ${ }^{9-16,21,22}$. In this regard, trilayer graphene is an attractive material system. Unlike bilayer graphene, however, trilayers, which typically exhibit Bernal (ABA) stacking order and the associated mirror symmetry (Fig. 1a), have been shown both theoretically ${ }^{9-16}$ and experimentally ${ }^{8}$ not to support the induction of a significant band gap when subjected to a perpendicular electric field. As discussed below, this behaviour follows from the mirror symmetry of the unperturbed ABA trilayer ${ }^{10,23}$. Recent research ${ }^{24,25}$ has, however, reported the existence of a new type of trilayer graphene, one with ABC (rhombohedral) stacking order between the graphene sheets (Fig. 1b). This crystal structure, like that of the bilayer, possesses inversion symmetry, but lacks mirror symmetry (Fig. 1b). The low-energy electronic structure of the $\mathrm{ABC}$ trilayer $^{20,22}$ is accordingly more similar to that of the $\mathrm{AB}$-stacked bilayer graphene. In particular, the undoped $\mathrm{ABC}$ trilayer has only two-fold degeneracy ${ }^{20}$ at the Fermi energy, like the graphene bilayer, rather than the four-fold degeneracy found in the ABA trilayer ${ }^{20,23}$. The two-fold degeneracy in the $\mathrm{ABC}$ trilayer band structure can be readily lifted by imposing different potentials on the top and bottom graphene layers by an applied electric field, which leads to the opening of a band gap $9,10,13-16,21,22$. Although theory has predicted the induction of a large band gap for $\mathrm{ABC}$ trilayer graphene, experimental confirmation has been lacking.
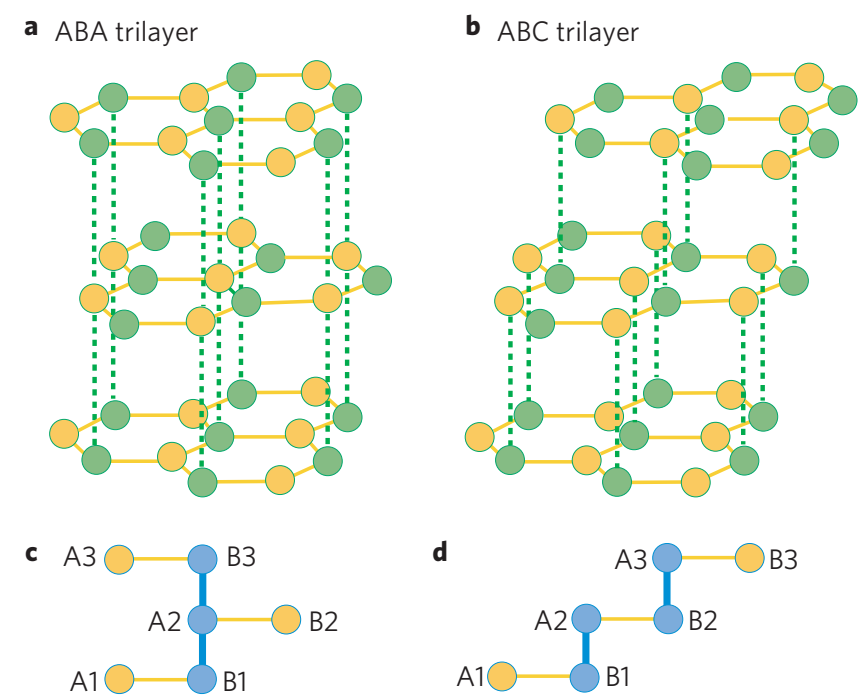

Figure 1 | Crystal structure and tight-binding diagrams for trilayer graphene with $A B A$ and $A B C$ stacking order. $\mathbf{a}, \mathbf{b}$, Crystal structure of $A B A(\mathbf{a})$ and $A B C(\mathbf{b})$ trilayer graphene. The yellow and green dots represent the $A$ and $B$ sublattices of the graphene honeycomb structure. $\mathbf{c}, \mathbf{d}$, Tight-binding diagrams for ABA (c) and ABC (d) trilayer graphene. At the K-point, the effective intralayer coupling vanishes. The atoms in yellow then become non-bonding monomers and the atoms in blue form a trimer in the $A B A$ trilayer and two dimers in the $A B C$ trilayer.

In this paper, we report an experimental and theoretical study of the electronic response of trilayer graphene, both of $\mathrm{ABA}$ and $\mathrm{ABC}$ stacking order, to perpendicular electric fields as strong as $\sim 0.3 \mathrm{~V} \mathrm{~nm}^{-1}$. Our results provide direct spectroscopic signatures of the induction of a tunable band gap of as much as $\sim 120 \mathrm{meV}$ in $\mathrm{ABC}$ trilayer graphene. Such a band gap is not observable in ABA trilayers under the same electric field. We analyse these results by considering the implications of the different crystal structure and interlayer coupling in ABA- and ABC-stacked trilayers.

We investigated graphene trilayer samples exfoliated from kish graphite on $\mathrm{SiO}_{2} / \mathrm{Si}$ substrates. The sample thickness and stacking order were first determined by infrared ${ }^{24-27}$ and Raman ${ }^{25}$ spectroscopy. In our measurements, we made use of an electrolyte top gate ${ }^{5}$ (Fig. 2a) to induce high doping densities and electric fields in the samples. The resultant change of the band structure was probed by infrared conductivity measurements (see Methods).

We have measured the infrared sheet conductivity $\sigma(\hbar \omega)$ of $\mathrm{ABA}$ and $\mathrm{ABC}$ trilayer graphene samples at different gate voltages $V_{\mathrm{g}}$ (Fig. 2). At the charge neutrality point $\left(V_{\mathrm{g}}=V_{\mathrm{CN}}\right)$,

\footnotetext{
${ }^{1}$ Departments of Physics and Electrical Engineering, Columbia University, 538 West 120th Street, New York, New York 10027, USA, ${ }^{2}$ Institute for Complex Systems (ISC), CNR, via dei Taurini 19, 00185 Rome, Italy, ${ }^{3}$ Instituto de Ciencia de Materiales de Madrid, CSIC, 28049 Cantoblanco, Madrid, Spain. *e-mail: tony.heinz@columbia.edu.
} 
a
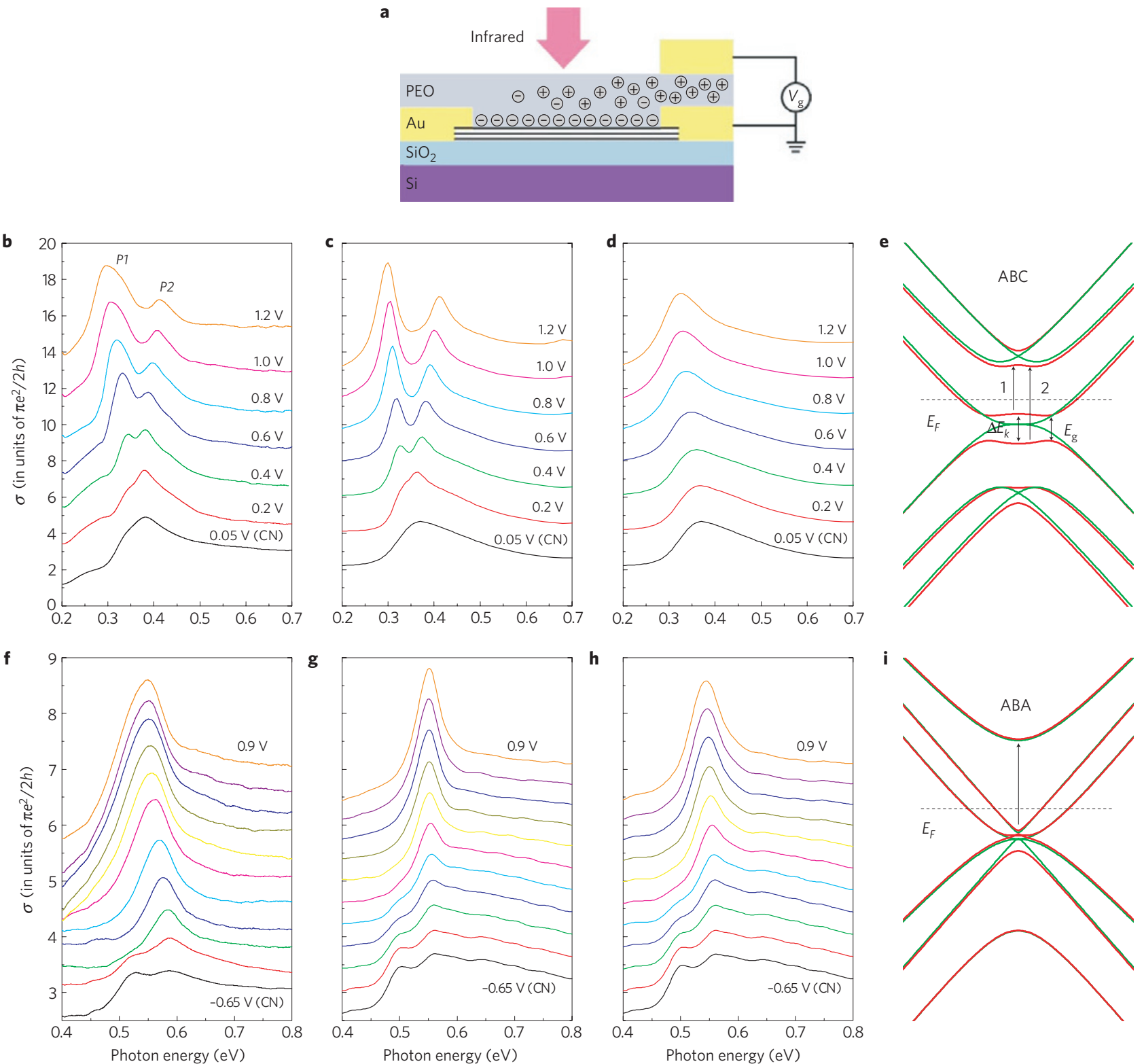

Figure 2 | Comparison of optical conductivity $\sigma(\hbar \omega)$ of $A B A$ and $A B C$ graphene trilayers for different gate voltages $V_{g}$. a, Schematic representation of the trilayer device used in these studies and described in the Methods section. $\mathbf{b}$, Experimental results for the gate-dependent optical conductivity spectra $\sigma(\hbar \omega)$ of ABC-stacked trilayer graphene. $\mathbf{c}, \mathbf{d}$, Theoretical simulations of $\sigma(\hbar \omega)$ for ABC-stacked trilayer graphene under the same gating conditions as in $\mathbf{b}$ c shows the predictions of the TB model for the electronic structure described in the text, whereas $\mathbf{d}$ is a reference calculation in which the band structure is assumed to remain unaltered with gating and only the induced population changes are taken into account. In $\mathbf{b}$-d, the gate voltages $V_{g}$ and the condition of charge neutrality $\left(V_{g}=V_{C N}=-0.65 \mathrm{~V}\right)$ are denoted on the spectra, which are displaced from one another by 2 units for clarity. e, The predicted band structure of $A B C$ trilayer graphene with (red) and without (green) the presence of a perpendicular electric field, as calculated within the TB model described in the text. Transitions 1 and 2 are the strongest optical transitions near the K-point for electron doping. f-h, Results corresponding to $\mathbf{b}$-d, for ABA-stacked trilayer graphene samples. The different spectra, from top to bottom, were obtained for gate voltages $V_{g}=0.9,0.7,0.5,0.3,0.1,-0.1,-0.3$, $-0.4,-0.5,-0.6,-0.65(\mathrm{CN}) \vee$ and are displaced from one another by 0.4 units. $\mathbf{i}$, The predicted band structure of ABA trilayer graphene with (red) and without (green) a perpendicular electric field, as calculated within the TB model described in the text. The arrow indicates the transition responsible for the main absorption peak at $0.5-0.6 \mathrm{eV}$.

the $\mathrm{ABC}$ spectrum shows a single absorption peak at $\hbar \omega=0.35 \mathrm{eV}$ (Fig. 2b), and the ABA spectrum exhibits two peaks at 0.52 and $0.585 \mathrm{eV}$ (Fig. 2f). These transitions reflect the distinct nature of the interlayer interactions and low-energy band structure (Fig. 2e,i) for the two types of crystal structures. The energies of the absorption peaks in $\mathrm{ABC}$ and $\mathrm{ABA}$ trilayer graphene correspond approximately to $\gamma_{1}$ and $\sqrt{ } 2 \gamma_{1}$, respectively, where $\gamma_{1} \sim 0.37 \mathrm{eV}$ is the nearest-neighbour interlayer coupling strength.
The factor of $\sqrt{ } 2$ arises from the fact that the atoms in the middle layer of the ABA structure are coupled symmetrically with atoms in both the bottom and top layers (Fig. 1a,c). We note that the two slightly different transition energies of 0.52 and $0.585 \mathrm{eV}$ in $\mathrm{ABA}$ trilayer graphene correspond, respectively, to hole and electron transitions ${ }^{5,28,29}$. (See Supplementary Information for more detailed analysis of the electron-hole asymmetry in ABA trilayer graphene.) 
As we increase the gate bias for the $\mathrm{ABC}$ trilayer, the main peak splits into two distinct features ( $P 1$ and $P 2$ in Fig. 2b) that shift in opposite directions and broaden. This behaviour is a clear signature of the induction of a band gap. Corresponding effects are also observed when a negative gate voltage is applied to produce hole doping (as described in the Supplementary Information). Figure 2e shows the evolution of the electronic structure of $\mathrm{ABC}$ trilayer graphene under an applied electric field according to a tight-binding (TB) calculation that includes the dominant intralayer $\left(\gamma_{0}\right)$ and interlayer $\left(\gamma_{1}\right)$ couplings. The unperturbed ABC trilayer (green line) has three valence and conduction bands near the K-point in the Brillouin zone. The two low-energy bands touch at the K-point, whereas the other bands are separated by $\gamma_{1} \sim 370 \mathrm{meV}$. With the application of a strong electric field, a gap develops between the low-energy valence band and conduction band (red line). The observed absorption peaks $P 1$ and $P 2$ are readily understood as arising from the transitions indicated as 1 and 2 in the modified band structure. The difference between $P 1$ and $P 2$ hence reflects the size of the band gap, which reaches $\sim 120 \mathrm{meV}$ at the highest applied gate voltage of $1.2 \mathrm{~V}$.

For the ABA trilayer, as we increase the gate bias, the amplitude of the transition at $0.585 \mathrm{eV}$ grows and the peak position redshifts, whereas the low-energy peak at $0.520 \mathrm{eV}$ disappears (Fig. 2f). A similar effect was observed for negative gate biases and hole doping (see Supplementary Information). Apart from state-filling effects that reflect the increase of Fermi level under gating, there is no evidence of the emergence of additional peaks associated with the creation of a band gap. We estimate from the broadening of the absorption peak that an induced band gap, if it exists, should not exceed $30 \mathrm{meV}$ at the highest gating voltage of $0.9 \mathrm{~V}$.

The above observations can be understood within a framework of the TB description, with a self-consistent scheme ${ }^{11}$ to take into account the gate-induced electric field across the graphene layers (see Methods). For the ABC case, we considered only the dominant coupling terms of $\gamma_{0}$ and $\gamma_{1}$. Carrying out the TB calculation with a full set of coupling parameters did not yield significantly different predictions. To obtain the best fit to the data, we used a value for the interlayer coupling of $\gamma_{1}=377 \mathrm{meV}$ and assumed a capacitance of the electrolyte top gate of $C_{\mathrm{g}}=1.3 \mu \mathrm{F} \mathrm{cm}^{-2}$. The predicted band gap, $E_{\mathrm{g}}$, and the energy gap at the K-point, $\Delta E_{\mathrm{k}}$, agree well with the band gap extracted from experiment (Fig. 3). For more detailed and direct comparison, we calculated the expected infrared conductivity spectra by means of the Kubo formula (Fig. 2c). These simulations clearly reproduce the main features of the experimental spectra (Fig. 2b). We also show for comparison the predicted conductivity when neglecting any induced modification of the electronic structure or band gap opening (Fig. 2d), including only the effect of state filling on the optical transitions. The resulting behaviour is completely inconsistent with experiment.

In the case of the ABA trilayer structure, we included in the TB simulations parameters that describe the observed electron-hole asymmetry. In particular, we used $\delta=37 \mathrm{meV}$ as the average on-site energy difference between atomic sites A1, B2, A3 and B1, A2, B3 (Fig. 1c) and $v_{4} \equiv \gamma_{4} / \gamma_{0}=0.05$ to describe the next-nearestneighbour interlayer coupling strength. We found reasonable agreement between the experiment and the $\sigma(\hbar \omega)$ spectra simulated by the Kubo formula (Fig. $2 \mathrm{~g}$ ) using parameters $\left(\gamma_{1}=371 \mathrm{meV}\right.$ and $C_{\mathrm{g}}=0.8 \mu \mathrm{F} \mathrm{cm}^{-2}$ ) similar to those for ABC stacking. For comparison, we also show the calculated $\sigma(\hbar \omega)$ spectra when neglecting any induced modification of the band structure (Fig. $2 \mathrm{~h}$ ). The resultant spectra are rather similar to the previous simulations (Fig. $2 \mathrm{~g}$ ). This conclusion is consistent with a predicted band structure for the ABA trilayer that changes little under the applied electric field (Fig. 2i).

As this analysis shows, the induction of a band gap in graphene trilayers is completely different for $\mathrm{ABA}$ - and $\mathrm{ABC}$-stacked materials. For applied electric fields of similar strength, the $A B C$

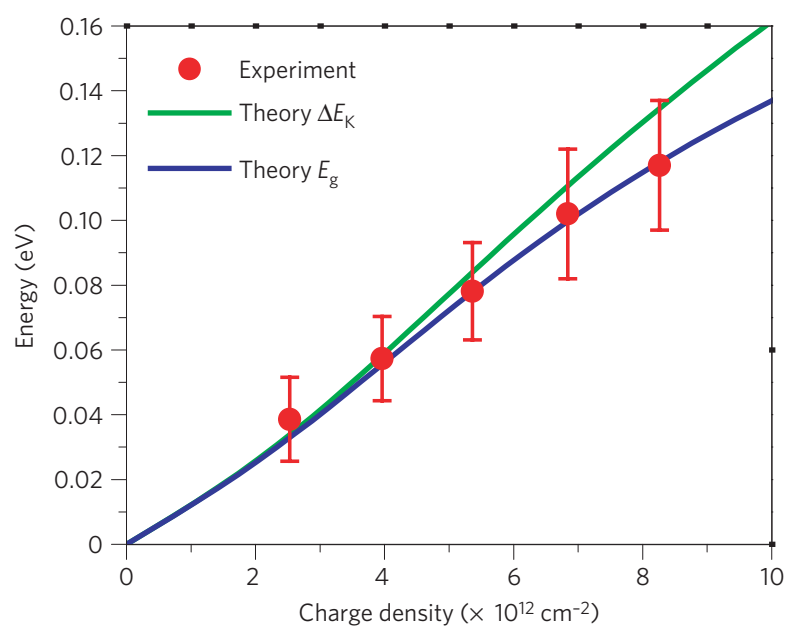

Figure 3 | Dependence of the energy gap on the induced charge doping density for $A B C$ trilayer graphene. The symbols are experimental data. The error bars arise primarily from uncertainties in determining the peak position of the absorption features. The results of the TB model for both the gap at the K-point $\Delta E_{K}$ (green line) and the band gap $E_{g}$ (blue line) are plotted for comparison.

trilayer shows a sizable band gap of $\sim 120 \mathrm{meV}$, whereas the ABA trilayer does not exhibit any signature of band-gap opening. The different behaviour can be understood within a TB model ${ }^{10}$ using only the dominant intra- and interlayer parameters of $\gamma_{0}$ and $\gamma_{1}$ (Fig. 1c,d). At the K-point of the Brillouin zone, the effective intralayer coupling vanishes. The states of $\mathrm{ABC}$ trilayer graphene can hence be represented by two dimers with finite energies $\left( \pm \gamma_{1}\right)$ and two monomers with zero energy (blue and yellow atoms in Fig. 1d, respectively). The application of a perpendicular electric field leads to different potentials at the bottom and top layers. This lifts the degeneracy of the two corresponding monomer states (A1 and B3) and induces a band gap. On the other hand, the electronic states at the K-point in the ABA trilayer system are represented by one trimer and three monomers (blue and yellow atoms in Fig. 1c, respectively). The trimer has a non-bonding state that forms a fourfold degenerate zero-energy level with the monomers. Although a vertical electric field can lift the degeneracy of the two monomer states on the bottom and top layers (A1 and A3), it has no appreciable influence on the monomer state on the middle layer (B2) and the non-bonding trimer state. The presence of this remaining degeneracy precludes the induction of a band gap in the ABA trilayer.

It is interesting to compare our results with the behaviour found in bilayer graphene under the influence of an applied electric field $^{4-6}$. An increased sensitivity to an applied electric field is expected for ABC-stacked trilayer graphene samples because the size of the induced band gap for a given field increases with layer thickness. In particular, for the same (moderate) applied field, the band gap in the thicker ABC trilayer should be approximately twice as large as in the $\mathrm{AB}$ bilayer. Experimentally, however, we have observed comparable band gaps for the two cases under similar applied electric fields. The disagreement may reflect the presence of nonlinear screening effects in the ABC-stacked trilayer graphene ${ }^{13}$. In view of the relatively poor calibration of the absolute strength of the applied electric fields, more controlled measurements are needed for complete clarification of this point. We note that for applications involving a material with tunable infrared properties, we found that the infrared peaks in $\mathrm{ABC}$ trilayers are much sharper than those observed in bilayers ${ }^{5}$ because of the higher-order van Hove singularity in the ABC trilayer band structure ${ }^{20,22}$. These better-defined features favour trilayers for applications requiring a tunable change in infrared absorption. More generally, our work 
suggests that a tunable band gap can be induced in thicker graphene samples with ABC (rhombohedral) stacking order ${ }^{24,25,30}$, thus providing a still broader class of materials with a tunable band gap.

\section{Methods}

Sample preparation and characterization. Graphene trilayer samples were prepared by mechanical exfoliation of kish graphite (Toshiba) on silicon substrates coated with a 300-nm oxide layer. The sample thickness and stacking order were characterized by means of infrared spectroscopy ${ }^{24-27}$ using the National Synchrotron Light Source at Brookhaven National Laboratory (U12IR beam line). For a more detailed analysis of the spatial variation of the sample, we relied on scanning Raman spectroscopy ${ }^{25}$. Using the signature of the stacking order in the 2D Raman feature, we could visualize the spatial distribution of the ABA and $\mathrm{ABC}$ stacking domains in trilayer samples. We found $\sim 60 \%$ of trilayer samples were of purely $\mathrm{ABA}$ stacking order, whereas the rest exhibited mixed ABA-ABC stacking orders ${ }^{25}$. For our investigations, we chose for device fabrications those samples showing either pure ABA stacking or large $\left(>200 \mu \mathrm{m}^{2}\right)$ homogeneous domains of $\mathrm{ABC}$ stacking.

Device fabrication. The graphene device structure used top gating of the graphene trilayer sample with a polymer electrolyte gate (Fig. 2a). Electrical contacts to the graphene samples were formed using electron-beam lithography and electron-beam evaporation of Au films of 50-nm thickness. The polymer electrolyte (poly(ethylene oxide) (PEO): $\mathrm{LiClO}_{4}, 8: 1$, dissolved in methanol) ${ }^{5}$ was then cast onto the sample and dried at $110^{\circ} \mathrm{C}$ in ambient. A large Au electrode, deposited within $100 \mu \mathrm{m}$ of the graphene samples, provided electrical contact to the transparent polymer gate. The capacitance of such top gates was typically $\sim 1.0 \mu \mathrm{F} \mathrm{cm}^{-2}$ and thus allowed us to induce charge densities of $\sim 10^{13} \mathrm{~cm}^{-2}$.

Determination of the optical conductivity. We measured the infrared transmission spectrum of the gated trilayers by normalizing the sample spectrum with that from the bare substrate. We then extracted the real part of the optical sheet conductivity $(\sigma)$ in the spectral range $0.2-1.0 \mathrm{eV}$ from the transmission spectra by solving the optical problem for a thin film on the $\mathrm{SiO}_{2}(300-\mathrm{nm}) / \mathrm{Si}$ substrate. In our calculation, we omitted the interference from the sample/PEO interface and considered only the much stronger reflection from the $\mathrm{SiO}_{2} / \mathrm{Si}$ interface. We also neglected the contribution of the imaginary part of the optical conductivity. The above simplifications are estimated to induce $10 \%$ errors in $\sigma$, mainly in the spectral range below $0.3 \mathrm{eV}$, and have negligible influence on the spectral positions of the peaks in $\sigma$.

Theoretical simulation of the optical conductivity. We applied the self-consistent approach of Avetisyan et al. ${ }^{11}$ to calculate the charge density for each layer of the graphene trilayers for different total charge density. In the calculation, we considered only the dominant $\gamma_{0}$ and $\gamma_{1}$ couplings in the $\mathrm{ABC}$ trilayer $\mathrm{TB}$ Hamiltonian and $\gamma_{0}, \gamma_{1}, \gamma_{3}$ and $\delta$ in the ABA trilayer TB Hamiltonian. We used the dielectric constant of bulk graphite $(\kappa=2.4)$ in the calculation. With the calculated self-consistent charge distribution, we simulated the optical conductivity using the Kubo formula with a broadening parameter of $10 \mathrm{meV}$.

Received 3 May 2011; accepted 19 August 2011; published online 25 September 2011

\section{References}

1. Ohta, T., Bostwick, A., Seyller, T., Horn, K. \& Rotenberg, E. Controlling the electronic structure of bilayer graphene. Science 313, 951-954 (2006).

2. Castro, E. V. et al. Biased bilayer graphene: Semiconductor with a gap tunable by the electric field effect. Phys. Rev. Lett. 99, 216802 (2007).

3. Oostinga, J. B., Heersche, H. B., Liu, X. L., Morpurgo, A. F. \& Vandersypen, L. M. K. Gate-induced insulating state in bilayer graphene devices. Nature Mater. 7, 151-157 (2008).

4. Zhang, Y. B. et al. Direct observation of a widely tunable bandgap in bilayer graphene. Nature 459, 820-823 (2009).

5. Mak, K. F., Lui, C. H., Shan, J. \& Heinz, T. F. Observation of an electric-field-induced band gap in bilayer graphene by infrared spectroscopy. Phys. Rev. Lett. 102, 256405 (2009).

6. Kuzmenko, A. B., Crassee, I., van der Marel, D., Blake, P. \& Novoselov, K. S. Determination of the gate-tunable band gap and tight-binding parameters in bilayer graphene using infrared spectroscopy. Phys. Rev. B 80, 165406 (2009)

7. Xia, F. N., Farmer, D. B., Lin, Y. M. \& Avouris, P. Graphene field-effect transistors with high on/off current ratio and large transport band gap at room temperature. Nano Lett. 10, 715-718 (2010).
8. Craciun, M. F. et al. Trilayer graphene is a semimetal with a gate-tunable band overlap. Nature Nanotech. 4, 383-388 (2009).

9. Guinea, F., Neto, A. H. C. \& Peres, N. M. R. Electronic states and Landau levels in graphene stacks. Phys. Rev. B 73, 245426 (2006).

10. Aoki, M. \& Amawashi, H. Dependence of band structures on stacking and field in layered graphene. Solid State Commun. 142, 123-127 (2007).

11. Avetisyan, A. A., Partoens, B. \& Peeters, F. M. Electric field tuning of the band gap in graphene multilayers. Phys. Rev. B 79, 035421 (2009).

12. Avetisyan, A. A., Partoens, B. \& Peeters, F. M. Electric-field control of the band gap and Fermi energy in graphene multilayers by top and back gates. Phys. Rev. B 80, 195401 (2009).

13. Koshino, $M$. Interlayer screening effect in graphene multilayers with $A B A$ and ABC stacking. Phys. Rev. B 81, 125304 (2010).

14. Kumar, S. B. \& Guo, J. Multilayer graphene under vertical electric field. Appl. Phys. Lett. 98, 222101 (2011).

15. Wu, B. R. Field modulation of the electronic structure of trilayer graphene. Appl. Phys. Lett. 98, 263107 (2011).

16. Tang, K. et al. Electric-field-induced energy gap in few-layer graphene. J. Phys. Chem. C 115, 9458-9464 (2011).

17. Zhou, S. Y. et al. Substrate-induced bandgap opening in epitaxial graphene. Nature Mater. 6, 916 (2007)

18. Balog, R. et al. Bandgap opening in graphene induced by patterned hydrogen adsorption. Nature Mater. 9, 315-319 (2010).

19. Latil, S. \& Henrard, L. Charge carriers in few-layer graphene films. Phys. Rev. Lett. 97, 036803 (2006).

20. Min, H. K. \& MacDonald, A. H. Electronic structure of multilayer graphene. Prog. Theor. Phys. Suppl. 176, 227-252 (2008).

21. Avetisyan, A. A., Partoens, B. \& Peeters, F. M. Stacking order dependent electric field tuning of the band gap in graphene multilayers. Phys. Rev. B 81, 115432 (2010).

22. Zhang, F., Sahu, B., Min, H. \& MacDonald, A. H. Band structure of ABC -stacked graphene trilayers. Phys. Rev. B 82, 035409 (2010).

23. Koshino, M. \& McCann, E. Gate-induced interlayer asymmetry in ABA-stacked trilayer graphene. Phys. Rev. B 79, 125443 (2009).

24. Mak, K. F., Shan, J. \& Heinz, T. F. Electronic structure of few-layer graphene: Experimental demonstration of strong dependence on stacking sequence. Phys. Rev. Lett. 104, 176404 (2010).

25. Lui, C. H. et al. Imaging stacking order in few-layer graphene. Nano Lett. 11, 164-169 (2010).

26. Mak, K. F., Sfeir, M. Y., Misewich, J. A. \& Heinz, T. F. The evolution of electronic structure in few-layer graphene revealed by optical spectroscopy. Proc. Natl Acad. Sci. USA 107, 14999 (2010).

27. Mak, K. F. et al. Measurement of the optical conductivity of graphene. Phys. Rev. Lett. 101, 196405 (2008).

28. Li, Z. Q. et al. Band structure asymmetry of bilayer graphene revealed by infrared spectroscopy. Phys. Rev. Lett. 102, 037403 (2009).

29. Kuzmenko, A. B. et al. Infrared spectroscopy of electronic bands in bilayer graphene. Phys. Rev. B 79, 115441 (2009).

30. Norimatsu, W. \& Kusunoki, M. Selective formation of ABC-stacked graphene layers on $\mathrm{SiC}(0001)$. Phys. Rev. B 81, 161410 (2010).

\section{Acknowledgements}

We thank G. L. Carr and R. Smith for technical support in the infrared measurement at Brookhaven National Laboratory, D. Efetov for support in device fabrication, and A. A. Avetisyan, B. Partoens, F. M. Peeters, M. Koshino, and Y. L. Li for discussions. The authors acknowledge support from the Office of Naval Research under the MURI program for device preparation and from the US Department of Energy under Energy Frontier Research Center grant DE-SC0001085 for spectroscopic measurements and analysis. E.C. acknowledges support from the European FP7 Marie Curie project PIEF-GA-2009-251904.

\section{Author contributions}

C.H.L. and Z.L. fabricated and characterized the samples, and carried out the measurements. K.F.M. led the design of the experiment and analysis methods. E.C., K.F.M. and C.H.L. developed the theoretical treatment and performed the simulations. All authors discussed the experiment and analysis. C.H.L. and T.F.H. wrote the manuscript.

\section{Additional information}

The authors declare no competing financial interests. Supplementary information accompanies this paper on www.nature.com/naturephysics. Reprints and permissions information is available online at http://www.nature.com/reprints. Correspondence and requests for materials should be addressed to T.F.H. 